\title{
A Method of Data Transfer Control during Handoffs in Mobile Multimedia Networks.
}

\author{
P.Venkataram, S.Laxman* and R.Rajavelsamy \\ Protocol Engineering and Technology Unit \\ Electrical Communication Engineering Department \\ Indian Institute of Science, Bangalore- 560012 \\ E-mail: pallapa@ece.iisc.ernet.in, \{laxman, rajvel\}@protocol.ece.iisc.ernet.in
}

\begin{abstract}
We propose a method to maintain the continuity of data transfer during handoffs in mobile networks. The method performs effcient buffering of the data at the Base station using toggled buffers. The method has been tested on in-house wired/wireless networks. The result have showed enormous improvement in the continuity of data transfer during multiple handoffs while running multimedia applications such as video-on-demand(VoD) and Audio-ondemand $(A o D)$.
\end{abstract}

Keywords: Buffering, Handoff, Mobile Network, Multimedia

\section{INTRODUCTION}

A key characteristics of multimedia type application services is that they require maintaining the continuity of data transfer. The population of the mobile users is growing at a rapid rate and that the applications are becoming more bandwidth intensive, hence the traffic control of wireless networks has become a necessary element of mobile communications. Due to the limitations of the radio spectrum, future wireless systems will use micro/picocellular architectures in order to provide a higher capacity. Because of the small coverage area of micro/pico-cells and the characteristics of the multipath and shadow fading radio environment. handoff events in future systems will occur at a much higher rate as compared to today's macro-cellular systems. Frequent handoffs in wireless/mobile networks introduce a new paradigm in the area of network congestion control. In future mobile networks are expected to support multimedia applications (video, audio, data, etc.), which will be an added challenge for designing predictive and adaptive algorithms for continuous data transfer for isochronous applications.

In this paper, a new data transfer control scheme is proposed to provide high degree of data transfer for multimedia traffic carried in microcellular, picocellular, and high speed wireless networks. The proposed scheme combines data transfer control and adaptive buffer management scheme to guarantee at call level QoS requirement of a real-time or non-real-time multimedia applications. This will maximize the resource utilization and minimize the data loss.

*presently working as Member(Research Staff) in Central Research Laboratory, Bharat Electronics Limited, Bangalore-54.
MOBILE MULTIMEDIA NETWORKS

A Mobile Multimedia Network[2] consists of several cells where each cell comprising of multimedia servers and a base-station. The communication among the Base-stations could be through wired network. The base-stations could be PCs or workstations equipped with adaptors for wireless networking. The adaptor acts as a gateways for communicating to the nearby mobile hosts and wired networks.

Mobile hosts could be a PDA or LAPTOP equipped wireless adaptor card. Each base-station covers some part of geographical area. Network connectivity will be continuously maintained as users carrying variety of mobile hosts roam from one cell to another. The mobile hosts can access data from servers, intelligent peripherals and the other users (wireless/wired) of the network through the base-station agents (PC's) used to facilitate the mobile IP working.

\section{PROBLEMS IN MOBILE MULTTMEDIA WIRELESS NET- WORKS}

Contrary to wired networks, wireless ones have to cope with very specific effects:

- The risk of collision of different bursts of information transmitted in the same time slot and at the same frequency, but originating at different transmitters.

- The presence of multipath propagation, leading to fading (flat or frequency-selective) and time delay spread, which give rise to ISI (Inter Symbol Interference) can strongly increase the BER (bit error rate). The biggest challenge in WAN (Wide Area Network) environment is the large multipath spread (several microseconds) in conjunction with the mobility of the channel. The large multipath spread can cause excessive scheme, RNET (Radio Network), which is robust against existing Doppler Shifts and flat fading.

- The limited resources in the lower frequency bands which became worse as the mobile communications are used by more people and used data rate per user increases. 
Multimedia Service Requirements

A typical distributed multimedia environment, in which multiple, remotely located users participate in a joint work or design project demand for resource sharing, multimedia data integration, local intelligence and autonomy, graphical interfaces and vendor independence

A multimedia environment, presently, consists of applications accessing pagers, facsimile, answering machines, telephone lines, speech synthesis, and digital recording and playback. Its key contributions are the integration of multiple media into a cohesive nomadic information infrastructure and a graceful transition from desktop to nomadic locals. This integration is at the service and user interface levels.

\section{HANDOFF PROCEDURE}

There are three phases in a handoff procedure.

1. Measurements: The mobile host as well as the access point do several measurements continuously. For eg., the signal strength is one parameter which might be measured by both the terminal and the access point.

2. Decision: Based on the measurements taken, a decision is made as to whether a handoff is required. For eg., a decision to perform a handoff might be taken if the signal strength goes below a specified threshold.

3. Execution: The actual handoff of the terminal from one cell to another is done in this phase. There are essentially two sub-phases in the execution of the handoff.

- New Link establishment.

- Release of old link.

Types Of Handoff Functions

- Mobile Initiated handoff: In this type of handoff, the mobile host has to manage the handoff. That is, it takes the measurements on the downlink, processes them, takes the decision to do the handoff, decides the target base-station, etc.

- Mobile Evaluated handoff: This is similar to the mobile initiated handoff except that the decision to do the handoff lies with the network.

- Network Initiated handoff: In this type of handoff, the network manages the handoff, which includes taking measurements on the uplink, processing them, deciding to do the handoff, deciding the target base-station, etc.

- Mobile Assisted handoff: This is similar to the network initiated handoff, except that the mobile assists the network by taking measurements along the downlink and relaying them back to the network.

\section{Based On The Direction Of The Handoff Signaling}

Yet another way of classifying the handoffs is the direction of the handoff signalling.

- Forward Handoff: After the mobile terminal decides the cell to which it will make a handoff, it contacts the basestation controlling the cell. The new base-station initiates the handoff signaling to delink the mobile terminal from the old base-station. This is especially useful if the mobile terminal suddenly loses contact with the current base-station. This is referred to as forward handoff.

- Backward Handoff: After the mobile terminal decides the cell to which it attempts to make a handoff, it contacts the current base-station, which initiates the signaling to do the handoff to the new base-station. This is referred to as backward handoff.

\section{Handoff Latency}

1. The time required to effect the handoff should be appropriate for the rate of mobility of the mobile terminal. That is, the decision to do the handoff should be valid for the current position of the mobile terminal after the handoff is completed.

2. In the context of wireless ATM, the switching of the VCs from the old path to the new path completed very quickly, in order to minimize the interruption to the cell transport.

\section{Handoff Using Mobile IP}

The Mobile IP uses a packet encapsulation and redirection technique as shown in the figure 1. The Mobile IP scheme is built around two components namely, the home-agent and the foreignagent. The home-agent is the entity that maintains a database of the current location of all the mobile terminals under its control. When a mobile node moves away from the home region to another region, the home-agent updates the database to contain the IP address of the foreign-agent that is currently controlling the mobile terminal. When an IP packet is sent to the mobile node, it first reaches the home-agent. The home-agent encapsulates the IP packet within another IP packet with the current foreign-agent as the destination. When the foreign-agent receives the encapsulated IP packet, it removes the IP header information inserted by the home-agent and sends the IP packet to the mobile terminal. Though the path from the mobile terminal to the fixed nodes is optimal, this scheme does not result in an optimal path from the fixed host to the mobile terminal. Handoff is the process of passing the responsibility of communication connectivity from one basestation to another. The resulting handoff traffic is a function of many factors, such as the number of mobile hosts, the size of the wireless cells, the desired channel capacity and the MH migration of speed and direction. As the wireless cells size becomes smaller and smaller, signaling traffic as a result of handoffs can be substantial. Therefore there is a need to reduce this handoff traffic; so that more bandwidth can be devoted to data transfer. This calls for an efficient handoff mechanism. The base-station keeps track of the mobile by the relative signal strength received from the $\mathrm{MH}$. There are two thresholds, and initial and a final threshold, for both 
sending and receiving mobiles as shown in the figure 1 , into the realm of a base-station. These thresholds exist to prevent sudden spikes of signals. For example, if a signal is fading gradually over a pre-determined period of time, then it will pass through the first threshold drop, and then a lower threshold drop. This insures that the signal will not drop suddenly and then retum to its normal value. Assume that the signal has faded below the second threshold. The base-station interrogates the Markov model to determine the next most probable location which is mobile is likely to take. The first base-station now sends a message to the second basestation to reserve resources for the new user at the new location.

Resource allocation is held at the new location based on a predefined QOS requirement, and a timer is started at the new location. The purpose of the timer is avoid holding the resources for an indefinite period of time, in case the mobile never enters the realm of the new base-station. When the new base-station completes the allocation, its send an ACK to the old base-station. The new base-station is now waiting for the arrival of the mobile.

The new base-station is now monitoring for the arrival of the mobile. There are two thresholds to be overcome as in the case of the sending base-station, only they are the inverse thresholds. If the mobile signal rises above the initial receiving threshold and gradually rises to the final receiving threshold, the new basestation now owns the mobile. The constraints are that the resource allocation timer has not expired at the new base-station. Handoff is now ready to be accomplished between the two base-stations.

With this method there may be a point in time when the signal strength is about the same in both base-station areas. The policy here, is that the base-station which is monitoring the mobile will claim the mobile if the signal is above a certain pre-determined threshold. The mobile now belongs to the new base-station which signals the old base-station that it now is assuming ownership of the mobile. The old base-station must update its address pointers to point to the mobile's new location so that the packet destined for the mobile can be re-routed to the new location, as in the case or standard handoff procedures described above.

\section{DATA TRANSFER DISCONTINUTTY DURING THE HANDOFF}

Multimedia applications such as presentational or conversational require huge amount of data. In mobile networks, there would be maximum possibility of losing data due to mobility of the users and some other means. This causes inconvenience to the end users(mobile hosts) because of discontinuity of data transfer while handoff takes place. Loss of data would be more worst if multiple handoff takes place in sequence. For example, consider a video on demand service is being requested by a mobile host. The parameters of the services are : the video length to be transmitted is 100 minutes, disk access rate in the correspondent host $(\mathrm{CH})$ is $1.5 \mathrm{Mb} / \mathrm{s}$, total number of bits of video information is equal to 9 Gbps $(100 \mathrm{~min} * 1.5 \mathrm{Mb} / \mathrm{s})$. Let digitalised Video is split into, say, 100 parts requiring $9 \mathrm{Mb}$ space in RAM for each part. So, $9 \mathrm{Mb}$ is received at base-station from the $\mathrm{CH}$ as a first consignment. Let us assume that the base-station transfers at the rate of $1.5 \mathrm{Mb} / \mathrm{s}$ to the mobile host. So, it takes $6 \mathrm{sec}$ for transfering first consinment. The playout at the mobile host for the application is atleast after receiving $9 \mathrm{Mb}$ of data. Now, let us assume that the mobile host

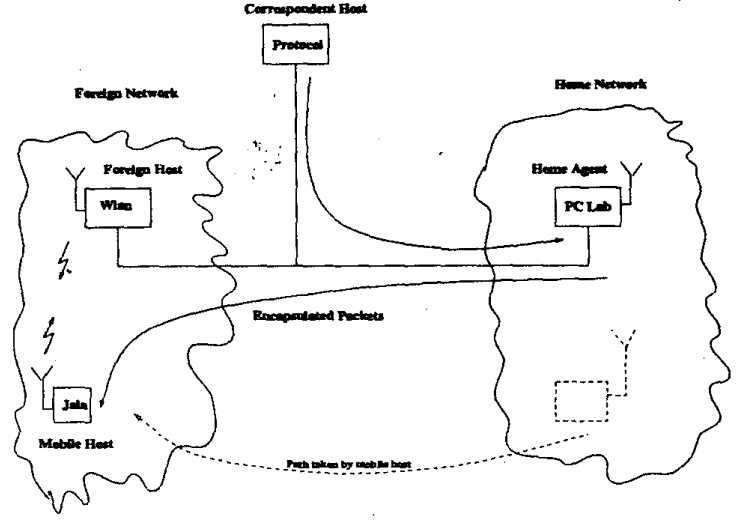

Figure 1: Handoff in Mobile IP

has initiated handoff call after running the application for $30 \mathrm{~min}$. The handoff period is normally two seconds. During this period, the amount of data transfered is $3 \mathrm{Mb}(2 \mathrm{sec} * 1.5 \mathrm{Mb} / \mathrm{s}$ ). The data transfered during this period will be lost. The video information in between $30^{\text {th }}$ minute and $32^{\text {th }}$ minute will not be received by mobile host. Thus there is discontinuity in the data received by the mobile host. This will deteriorate the quality of the application. Hence, it is essential to have a scheme for buffering the data during handoffs.

\section{PROPOSED DATA TRANSFER CONTROL}

The complete scenario of communication between HA (Basestation) and MH during the Handoffs can be explained as follows: the mobile host will move from home network to foreign network by intimating the home-agent about its mobility, so, that the homeagent will suspend the transmission of the packets which are destined to the mobile host until the registration process is completed with the foreign-agent; During this handoff the home agent will buffer the data in temporary buffer; After complete registration of mobile host with the foreign-agent, the home-agent will send the buffered data to the mobile host through the new foreign-agent.

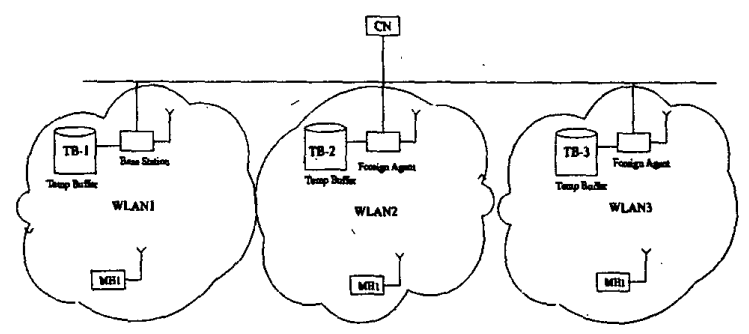

Figure 2: Proposed Data Transfer Control during Handoff in Mobile IP

For example, consider mobile agent given in figure 2. During data transfer session with the corresponding node (CN), MH1 has moved from WLAN1 to WLAN2 then to WLAN3. 


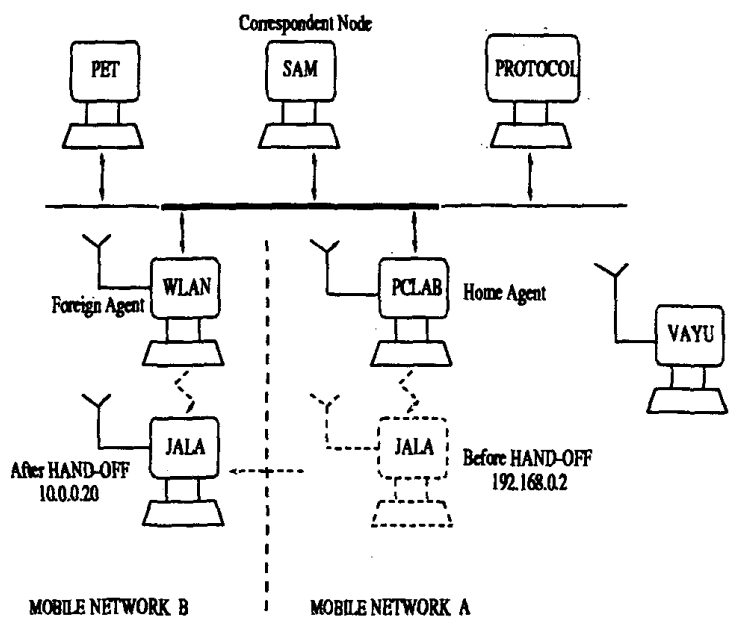

Figure 3: In-house wired/wireless Network Topology

When MH1 initiated Handoff in WLAN1, i.e., it is away from WLAN1 and its moving towards WLAN2, the data transfer to the MH1 is suspended and the received data being stored in the Temp Buffer (TB-1) in HA. When the handoff is completed the HA does tunneling, sends the data through TB-1 to the FA in WLAN2. Let the MH1 moves away from WLAN2 to WLAN3. During the Handoff data transfer to the $\mathrm{MH1}$ is suspended and the received data being stored in the Temp Buffer (TB-2). When the handoff is completed, the FA of WLAN2 does the tunneling and it sends the data from its Temp buffer(TB-2) to the FA in WLAN3.

So data transfer between the home-agent and mobile host or foreign agent is controlled using buffers to avoid the loss of data during handoff. The buffer size depends on the handoff period and the transfer rate at the base-station. Consider the example discussed in the previous section, we need a buffer space of $3 \mathrm{Mb}$ ( handoff period * transfer rate at the base-station) at the base-station.Thus the proposed method overcomes the problem of discontinuity of data transfer during the handoffs in mobile multimedia networks. However there are some overheads associated with this method. They are memory space, CPU time at the base-station. But these overheads will be nullified with respect to the achievement of continuous data transfer.

\section{SIMULATION MODEL}

we have simulated the proposed model of data transfer control and buffering data by configuring two wireless LANs[3].

Network Environment

We set up two wireless LANs(see figure 3) which are extended to exist Ethernet in our lab.

\section{Simulation Method}

We configured two mobile networks as shown in the figure 3 . The JALA (Pentium 100) is the MH, which will be running the mobile

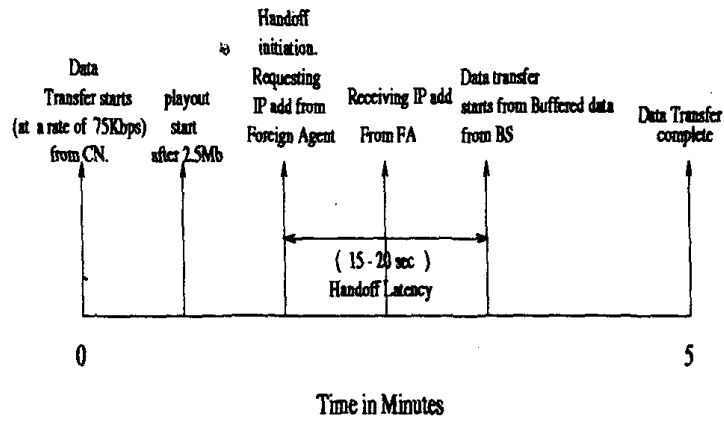

Figure 4: Timing of handoff events in the Handoff Process

host daemon. The $\mathrm{PC}$ lab (i486 66) is the home agent (HA), which will be running Home agent daemon. The mobile will initially be in its home network and will posses a long term IP address in its home. The WLAN (P 133) is configured as foreign-agent (FA). Since we have build up the system in such a way that the mobile hosts will get the new 'co-located care-of-address', WLAN will be running DHCP server. PROTOCOL (P 75) is the correspondent host (CH). This $\mathrm{CH}$ can be any hosts in the internet, in our case for some experiment the CH will be Sam ( SPARC 4).

We start all the daemons, home agent daemon at the PC lab. Mobile agent daemon at the JALA. This will register with the home agent. we started the DHCP server at WLAN. Now in order to initiate the movement of mobile host from its home-agent to any foreign network ( WLAN in this case) we will run the dhcp client at the mobile host (JALA), since only WLAN answers for dhcp request, it will provide a new IP address to JALA, thus JALA effectively is moved into foreign network ( WLAN network). Now based on the above setup, multimedia application was developed. The client ( the mobile host ) request for multimedia document ( may be a Mpeg file ) from the server, which will be a correspondent host. As the MPEG file is being played, the mobile host will initiate a handoff and moves to the foreign network ( WLAN network in our case ), the mpeg file is buffered at the base station for some time, during the process of registration, and later continues the mpeg file. The end user in the other hand will have see effect of the mobile host handoff but for a possible pause in the play without any data lose. Now in order to reduce or eliminate this pause a scheme was developed. This is a simple scheme, looking at the network traffic and type of the multimedia document, a predefined size of the document is buffered (the value is heuristically defined) So the application on the client side will start only after this minimum buffer is filled. All our experiments are conducted with minimum buffer criteria i.e., the playout starts after receiving the data around $2.5 \mathrm{Mb}$

\section{RESULTS}

We have simulated for five different applications with occurance of multiple handoffs. The results are tabulated.

Table-I describes the data transfer activity for five multimedia applications: according to the file size $5 \mathrm{Mb}, 10 \mathrm{Mb}, 15 \mathrm{Mb}, 20 \mathrm{Mb}$, $25 \mathrm{Mb}$. in the Mobile Environment. For application say $15 \mathrm{Mb}$ file, Average number of Handoff ( in 10 Trails ) is 2, Average time 


\begin{tabular}{|c|c|c|c|}
\hline Fle Stete & $\begin{array}{l}\text { Avg Nomber } \\
\text { of Hendorf }\end{array}$ & $\begin{array}{c}\text { Avg Time Tutent } \\
\text { per Hendoff }\end{array}$ & $\begin{array}{c}\text { Avg Dasta Lost } \\
\text { per Handoff }\end{array}$ \\
\hline $5 \mathrm{MB}$ & 25 & $18.68 \mathrm{sec}$ & $137 \mathrm{Mb}$ \\
\hline loMb & 23 & $19.08 \mathrm{Sec}$ & $1.40 \mathrm{Mb}$ \\
\hline 15Mb & 2 & $19.80 \mathrm{sec}$ & $1 / 4 \mathrm{Mb}$ \\
\hline $20 \mathrm{Mb}$ & 21 & $18.57 \mathrm{sec}$ & $1.39 \mathrm{Mb}$ \\
\hline 25Mb & 18 & 19.55 sece & $135 \mathrm{Mb}$ \\
\hline
\end{tabular}

Table -1 Handoff statistics for VoD application

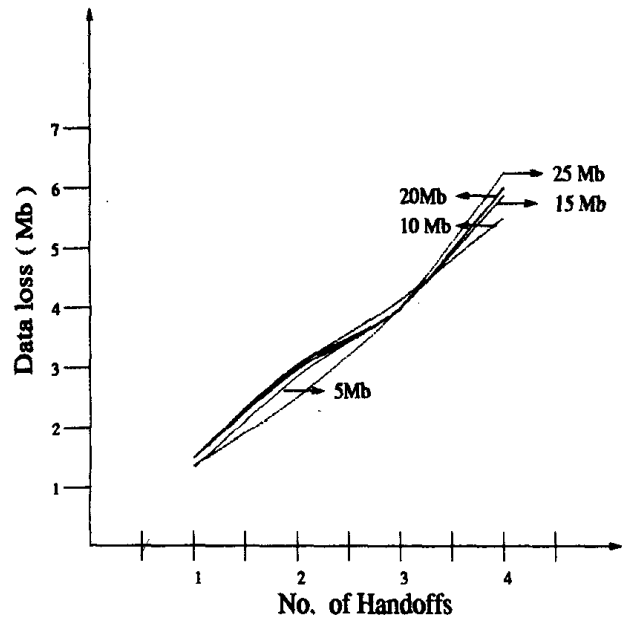

Figure 5: Handoff Vs Data Lost

for each Handoff is $19.8 \mathrm{Sec}$ in which Data lost per handoff is $1.45 \mathrm{Mb}$.

As number of handoffs increases data loss will be increased randomly with some positive amount, as shown in the graph above.

Table-II describes the results of experiments with and without buffering at the base station.

\section{CONCLUSION}

Real time mobile multimedia networks is challenging due to severe resource constraints of the wireless link and mobility of the links. The paper gives a brief introduction to mobile multimedia networks, multimedia service requirements, problems in mobile multimedia networks and mobile IP. One of the severe problem is the discontinuous data transfer during handoff which hinders the quality of application. We have proposed a method of continuous data transfer using buffering mechanism and simulated. It is observed through simulation that the buffer space increases with the increase in the number of handoffs. Also there was no loss

\begin{tabular}{|c|c|c|}
\hline Handoff Method & Handoff Latency & Averase Dath lost \\
\hline $\begin{array}{c}\text { Handoff without } \\
\text { Buffering }\end{array}$ & $15-20 \mathrm{Sec}$ & $139 \mathrm{Mb}$ \\
\hline $\begin{array}{c}\text { Handoff with } \\
\text { Buffering }\end{array}$ & $15-20 \mathrm{Sec}$ & $0.159 \mathrm{Mb}$ \\
\hline
\end{tabular}

Table - II Results of experiments with and without buffering during Handoff

of data during handoffs for different multimedia applications. However some overheads are associated with this mechanism such as buffer space and processing time.

\section{REFERENCES}

1. Venkataram $P$, Chockalingam $A$, and Roy $A$, "Performance of a Link Control Protocol for Local Wireless Multimedia Communications", IEEE Proc. of GLOBECOM Sydney, pp. 107-112, Nov. 8-12, 1998.

2. Venkataram $P, "$ Techniques for Achieving Highly Reliable and High Capacity in Multimedia Wireless Networks" Proc. of Intl. Symposium on Wireless Communications for the Next Millennium (ISWCM'98), pp. 122-130, Sept. 1998.

3. Lamaire RO, et. al, "Wireless LANs and Future Mobile Networking: Standards and Future Directions", IEEE Communication Magazine, pp. 86-94, Aug. 1996.

4. Chockalingam A, Zorzi, Milstein LB, and Venkataram $\mathbf{P}$, "Performance of a Wireless Access Protocol on Correlated Rayleigh Fading Channels with Capture",IEEE Trans. on Communications, Vol.46, No.5, pp 644-655, May 1998

5. Goodman DJ, Pollini GP, and Meier-Hellstern KS, "Network Control for Wireless Communications", IEEE Communication Magazine, pp. 116-124, Dec. 1992.

6. Perkins C, "IP Mobility Support, Internet Draft (draft-ietfmobilip-protocol-17.txt)", 1996.

7. Rappaport TS, Wireless Communications Principles and Practices, Prentice-Hall, Inc., New Jersey, 1996.

8. William C.Y. Lee, Mobile Cellular Telecommunications, Mc Graw-Hill Inc., 1996.

9. S. Seshan, H. Balakrishnan, R.H. Katz. " Handoffs in Cellular Wireless Networks: The Daedalus Implementation and Experience," Kluwer International Journal on Wireless Personal Communications, January 1997. 